when we have precisely the fourth exit, i.e., a position from which the defender can be stopped from resuming such a position.

As a postscript let me mention that psychologists have yet to agree on the nature of problem-solving difficulty, except that it is a speciality of humans. So when will the first psychologist realise that confrontations such as the Timman vs. Speelman ending offer opportunities for attacking precisely this question, using the appropriate extant databases as readymade infallible yardsticks?

\title{
ENDGAME SOURCE CODE GOES PUBLIC
}

\author{
Lewis Stiller \\ CNLS / MS B258 \\ Los Alamos National Laboratory \\ Los Alamos, NM 87545
}

The readers of this Journal may be interested in the fact that I have found a stable anonymous ftp site for the source code to create endgame databases. [See, e.g., ICCA Journal, Vol. 14, No. 3, pp. 129-134; Vol. 14, No. 4, pp. 198-201; Vol. 15, No. 1, pp. 16-18 for some of the recent results. - Eds.]

The anonymous ftp site is cmns.think.com (131.239.2.100) in archives/chess/snarc.public. I thank Thinking Machines Corporation for donating the ftp site.

I hope that this publicity encourages other implementations of my code (porting to other architectures).

\author{
Derek Oldbury \\ 4 Farm Close \\ Kingskerswell \\ Devon \\ England TQ12 5BT
}

ALPHA-BETA: A DISK MAGAZINE

The magazine's aim is to provide an informal link between the game-playing public and the specialised Societies which cater for the expert. This we do by publishing contact addresses of National and International groups; also, sources from which books and journals and equipment may be bought; also, a Calendar of important events. Increased membership of the various Associations is a prime target.

Additionally, we plan to run feature articles on the various games, to introduce the beauties of each game to players of other thinking games. We can include computer programs which play the game; arguably the best method of introducing a game to a newcomer, since it circumvents the need to purchase equipment before giving the game a trial! Where appropriate, we publish listings and discussions on how to program computer games - but this is about as far as we can go. We are not experts in all games. We need those dedicated groups and specialist Societies to contribute some interesting material (on disk, please), thus giving us a helping hand to propagate their activities. A reciprocal concept.

The project is still in its infancy. For those interested, the rates of subscription are: $\mathfrak{f 1 5}$ sterling per year (please add enough for bank charges if paying in a different currency), or $£ 4$ for a single issue. The magazine is published quarterly. 